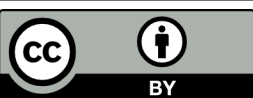

\title{
Musicoterapia y bienestar en personas adultas mexicanas
}

Javier Eduardo García de Alba García ${ }^{1}$

Ana Leticia Salcedo Rocha ${ }^{2}$

Judith Edith García de Alba Verduzco ${ }^{3}$

Ignacio Varela Flores ${ }^{4}$

Cristina Martínez Rocha ${ }^{5}$

Recibido: 10/07/2017 /Aceptado: 26/03/2018

\section{RESUMEN}

Objetivo: Determinar nivel de bienestar del adulto mayor mediante intervenciones musicales

Material y Métodos: Intervención azareada, doble ciega. En dos grupos con 15 mujeres, pertenecientes a un grupo de convivencia (educación y ejercicio).

Un grupo escuchó música, y otro música, con mensajes sobre salud integral. Se aplicó, antes y después de la intervención, cuestionario SF 36 de bienestar y salud percibidos. Se aplicaron estadígrafos descriptivos e inferenciales según escalas de las variables analizadas.

Resultados: Ambos grupos, presentaron variables sociodemográficas similares. $\mathrm{Y}$ al finalizar la intervención, mostraron en relación a la estimación inicial un incremento significativo de puntuaciones perceptivas en: salud general, funcionalidad social, rol emocional, y salud mental. Solo el grupo con mensajes, evidenció mejores puntaciones, en: dolor.

Conclusión: Los resultados, estimulan continuar y ampliar, el impacto de la musicoterapia en adultas mayores, posibilitando relacionar disciplinas científicas, con propósitos preventivos, terapéuticos y de rehabilitación para el bienestar del ser humano

Palabras Clave: Musicoterapia, Adultos mayores, SF36, Calidad de Vida, Promoción de la salud.

\section{Music therapy and well-being in Mexican adults}

\section{ABSTRACT}

Objective: To determine the level of well-being of the elderly through two interventions of music therapy

Material and methods: Double blind random intervention. In two groups with 15 persons, belonging to institutional attending educational and physical workouts. One Group listened to music, and another that music, accompanied by health-related messages. Questionnaire SF-36's of perceived well-being and health applied, before and after the intervention, The statistics analysis applied descriptive and inferential statisticians, in accordance with the analyzed variables.

\footnotetext{
${ }^{1}$ Investigador de la Unidad de Investigación Social, Epidemiológica y en Servicios de Salud- Instituto Mexicano del Seguro Social - Jalisco; javier.garciaal@imss.gob.mx

${ }^{2}$ Investigadora. Unidad de Investigación Social, Epidemiológica y en Servicios de Salud- Instituto Mexicano del Seguro Social - Jalisco; ana.salcedo@imss.gob.mx. Autora de la correspondencia

${ }^{3}$ Psicologa Especializada y responsable de Consejería y Acompañamiento Emocional. Unidad de Especialidades Médicas para la Detección y Diagnóstico del Cáncer de Mama de San Luis Potosí; judithgarciadealba@gmail.com

${ }^{4}$ Profesor. Biblioteca Ibero-Americana Universidad de Guadalajara. nachcvris@hotmail.com

${ }^{5}$ Licenciada en Biotecnología Universidad de Guadalajara.nachcvris@hotmail.com5
} 
Results: Both groups findings, presented similar socio-demographic results. At the end of the intervention, showed in relation to the pretest, significant increase of perceptual scores in: general health, social functioning, emotional role, and mental health. The group with oral messages, showed significant decrease, in: pain.

Conclusions: Results stimulate continue and expand, the impact of music therapy in older adults, making it possible to relate scientific disciplines, for the well-being of the human being

Key Words: Music Therapy, Older adults, SF36, Quality of Life, Health Promotion.

Sumario. 1.Introducción. 2.Material y métodos3. Resultados 4.Discusión 5. Conclusiones 6. Referencias

Cómo citar. García, J., Salcedo, A., García, J, Varela, I. y Martínez, C. (2018) Musicoterapia y bienestar en personas adultas mexicanas. Revista de Investigación en Musicoterapia, 2, 1-16

\section{Introducción}

El presente trabajo enfoca desde el paradigma cuantitativo la relación de una intervención musical con y sin información salutogéna, como, posibles herramientas complementarias del manejo integral para el bienestar de los adultos mayores.

En el caso de la música, y la palabra, además de su impacto físico, presentan un efecto emocional sobre los seres humanos, pues tiene la capacidad de integrar precisamente aspectos físicos, fisiológicos, emocionales, mentales y espirituales (Trailero. 2006).

La musicoterapia (MT) (Sanz.1989), como aplicación de la música en el tratamiento, la rehabilitación y el adiestramiento de personas que padecen trastornos físicos, mentales y emocionales, sus objetivos se relacionan con:

1.- Mejorar la afectividad, la conducta, la perceptiva motricidad, la personalidad y la comunicación (Myskja.2008).

2.- Mejorar las funciones psico-fisiológicas tales como el ritmo respiratorio y cardiaco, el trazo del electroencefalograma, la temperatura corporal, sensibilidad al dolor, y el restablecimiento de otros ritmos biológicos a través de la música.Benkovitz.2008),

Por otra parte la musicoterapia según Sanz (1989), ofrece al individuo tres experiencias a lograr:

1.- Experiencia dentro de una estructura, es decir durante la experiencia musical.

2.- Experiencia en la auto-organización, merced a respuestas personales a la conducta por la experiencia estética. 
3.- Experiencia en concordancia con los demás al relacionarse como miembros de un grupo.

Los mecanismos de acción atribuidos la musicoterapia, según sus técnicas son:

1) Técnicas Activas: cuyo mecanismo, es la acción terapéutica, para que la persona utilice su propia voz y/o cuerpo para estimularse.

2) Técnicas Receptivas: cuyo mecanismo de acción se atribuye a los estímulos musicales que inducen cambios emocionales y físicos.

Algunas de las experiencias publicadas, reportan muestras conformadas por un rango de 19 a 68 participantes, donde se ofrece música pregrabada, en sesiones de 60 a 90 minutos de duración, con periodicidad diaria, o de 3 veces por semana, durante 12 a 20 sesiones (Maratos, Gold, Wang, \&Crawford. 2008) y, se ha utilizado como coadyuvante para tratar diversas afecciones subjetivas y objetivas.

Estudios observacionales (Benezon.2000) concluyeron que se pueden tener efectos benéficos en personas con depresión. Al efecto, en un ensayo clínico (Redulovic, Cvetkovic, \& Pejovic.1997) controlado, auto administrado por los participantes, reporta al final del tratamiento una puntuación de 16.5 en el grupo activo, versus 25.1 en el grupo testigo $(\mathrm{p}<0.05)$.

Un estudio reciente (Shama.\& Mathur.2011), demostró diferencias estadísticamente significativas en un estudio controlado, a favor de un grupo con musicoterapia como coadyuvante para el control del síndrome metabólico en Bangladesh.

Asimismo se señala que existe una relación psicofisiológica entre el dolor y la música, como tratamiento complementario que puede lograr profundos niveles emocionales, particularmente efectiva en la etapa de cuidados paliativos, donde mejora la calidad de vida y amplifica el bienestar y la relajación. Además que el uso de la música para aliviar el dolor, ansiedad y depresión es cada vez más común. Mostrando evidencia en diferentes estudios para la modificación de niveles de dolor (Kopf, y Patel.2010). 
De acuerdo con lo antes señalado, la músico-terapia ha sido utilizada en el manejó de diversos trastornos, con resultados positivos, sin embargo poco ha sido relacionada con la calidad de vida, utilizando, instrumentos validados en nuestro contexto, por un equipo, donde había 2 médicos, 1 psicólogo, y 2 músicos.

\section{Objetivo}

General. Determinar calidad de vida percibida del adulto mayor, a través del cuestionario SF 36, en dos grupos,, antes y después de una experiencia musical con y sin mensajes salutogénos.

\section{Específicos}

- Realizar 22 sesiones musicales, con mensajes salutogénos durante un mínimo de 30 minutos, en uno de los grupos de adultos mayores participantes.

- Realizar 22 sesiones de musicales sin mensajes salutogénos durante un mínimo de 30 minutos, en uno de los grupos de adultos mayores participantes.

- Comparar en ambos grupos participantes los niveles de: Función física, Rol físico, Dolor corporal, Salud general, Vitalidad, Función Social, Rol emocional, y Salud mental.

\section{Material y Métodos}

Diseño del estudio. Intervención controlada con asignación aleatoria de la variable independiente con doble enmascaramiento.

El tamaño de la muestra, fue estimado basándonos en las puntuaciones máxima y mínima de calidad de vida relacionada a la salud, de los estudios nacionales reportados (Duran.2004), calculándose 15 personas, como número mínimo para detectar diferencias en las puntuaciones a evaluar con el instrumento SF 36, considerándose una confianza de $95 \%$ y un poder de $80 \%$.

El Universo de trabajo fue el grupo de convivencia "Dulces Amigas", con un total de 53 personas, que asiste en la Unidad de Investigación Social, Epidemiológica y Servicios de Salud (UISESS).”, que se subdividió al azar en dos, y aleatoriamente, se seleccionó, un grupo de 15 personas, para escuchar solo música, y en otro igual, para 
escuchar la misma música, acompañada de pequeños mensajes relacionados con la salud familiar, mental y física. Se incluyeron mujeres mayores de 60 años, con enfermedad crónica controlada, de estrato popular, residentes en el sector libertad de la ciudad de Guadalajara, que aceptaron participar, no se aceptaron pacientes clínicamente descompensadas o discapacitadas. No hubo deserciones.

\section{Variables de Estudio.}

\section{Variable independiente.}

Intervención con música. Variable cualitativa, de escala nominal, dicotómica. Para fines del estudio se considerará intervención con música, la exposición diaria, intima, durante 22 noches, por un mínimo de 30 minutos, con 6 melodías grabadas de música clásica, de ritmo tranquilo, en tono mayor, para propiciar un mejor estado de ánimo, es decir, buscar sentimientos de paz, tranquilidad, plenitud. Ya que las piezas debían ser escuchadas durante la noche, se evitaron instrumentos percutidos, incluido el piano. (Propicias para para inteligencia emocional), Las piezas seleccionada por dos de los investigadores, músicos profesionales, en su gran mayoría se emplearon piezas del barroco y Mozart cuyas formas musicales son más sencillas y "naturales". Autores como Beethoven, Chopin o Tchaikovski por ejemplo, se evitaron por ser demasiado apasionados u oscuros y melancólicos, emociones que queríamos evitar. Se seleccionaron Adagios y Larguetos con 58 a 60 negras por minuto, pues se buscó igualar la frecuencia de las ondas Alfa en el cerebro de alrededor de 60 ciclos por minuto: El grupo tuvo dos ramas activas: una con mensajes salutogénos entre cada melodía y otra sin mensajes salutogénos.

\section{Variable dependiente.}

Calidad de Vida. Variable cuantitativa de escala ordinal. Obtenida del puntaje obtenido del cuestionario SF36, con varias opciones de respuesta para 36 preguntas que se agrupan en 8 dominios.

\section{Variables intervinientes.}

Edad, Sexo y Pertenencia al grupo "Dulces Amigas" 


\section{Prevenciones y Limitantes del Estudio.}

1.-Para prevenir un sesgo de selección y de información, un investigador grabó el material de la intervención en memoria USB que identificó mediante un número aleatorio y entregó a otro investigador, quien a su vez lo proporcionó a los adultos participantes, asignándolo con ayuda de una tabla de números aleatorios, cuidando de cotejar el código del material y la identificación del participante. Otros investigadores, aplicaron el cuestionario SB 36 antes y después de la intervención.

2.- El carácter -exploratorio del estudio y su pequeño tamaño impide realizar generalizaciones de los resultados a otras poblaciones.

\section{Procedimientos e Instrumentación del Estudio.}

Parte 1.- Registro de las variables sociodemográficas (antes de la intervención)

Parte 2.- Registro de calidad de vida (antes y después de la intervención). A través del índice de abreviado de Calidad de Vida SF 36.

Intervención de música. De acuerdo con el método de experiencia receptiva, descrito por Bruscia.(2000) se aplicaron seis melodías seleccionadas para oírse durante un periodo mínimo de 30 minutos diariamente en casa antes de acostarse a dormir, de acuerdo a unas breves instrucciones previas.

El tempo de la música seleccionada fue de 60 compases por minuto, sin sonidos acentuados, percusiones o ritmos sincopados (Chan, Chan, Mok, Tse, \& Yuk. 2009).

Adicionalmente se llevó un cuaderno de registro de sesiones cumplidas y satisfacción obtenida.

Para la reproducción de la música se prestó un radio reproductor con entrada para USB, marca Súper Sonic ${ }^{\circledR}$ SC 1300K, de corriente eléctrica alterna (120 voltios) con y batería recargable de inicio automático para evitar pérdidas súbitas de energía.

La música editada para su audición, consistió en 6 melodías clásicas, de ritmo tranquilo (con y sin mensajes salutogénos relacionados con la salud familiar, física y mental) que se reprodujeron a partir de grabaciones originales, con un aparato de alta fidelidad, en memorias USB flash drive de 4 GB marca ADATA®. 
La instrucción fue escucharla a volumen bajo en todas las noches, durante 22 días seguidos.

Recolección de datos. Los datos técnicos de las partes 1, 2, del estudio, fueron colectados aplicando los instrumentos correspondientes, por los investigadores capacitados en el registro de la información.

Los datos de la aplicación de la intervención musical, previo adiestramiento personal, fueron auto registrados por los adultos mayores participantes, quienes en algunos casos, fueron apoyados por un familiar.

Proceso y análisis de datos. El proceso de los datos se efectuó con apoyo del programa SPSS 15.0 para elaborar una base de datos, la cual sirvió para realizar el análisis estadístico de tipo descriptivo e inferencial de acuerdo al tipo de escala utilizada.

Cronograma de actividades. El estudio desde su aprobación por los comités institucionales de ética e investigación, hasta su redacción final incluyo desde el verano de del año 2015 al verano del 2016.

Consideraciones éticas. Las intervenciones con musicoterapia no refieren reacciones secundarias o adversas (Myskja.2008). En el estudio se garantizó el respeto a los principios de: Autonomía en la participación, Justicia en el trato para los grupos participantes, y Beneficencia no maleficencia en la intervención,

De acuerdo a la Ley General de Salud (artículo 17, fracción 1), se clasifica el estudio como de riesgo mínimo, solicitándose consentimiento informado por escrito a los adultos mayores participantes.

La confidencialidad de la información estuvo garantizada al manejar las identidades personales mediante la asignación de claves para cada participante.

\section{Resultados}


El promedio edad del grupo con mensaje fue de $64.00 \pm 9.50$ años y grupo sin mensaje $61.93 \pm 7.68(\mathrm{p}=0.156)$

En la tabla 1, En el grupo con mensaje, las puntuaciones del cuestionario SF36 pre-post- intervención, evidencian diferencias estadísticamente significativas $(\mathrm{p}<0.05)$, en los puntajes reportados para cinco de las ocho áreas investigadas: dolor corporal, salud general, funcionamiento social, rol emocional y salud mental.

En la tabla 2, el análisis del grupo sin mensaje pre-post intervención, muestra diferencias estadísticas en seis de las ocho áreas: funcionalidad física, rol físico, vitalidad, rol emocional; y salud mental.

En la tabla 3, el análisis inter grupos, para el pretest, del cuestionario SB36, evidencia diferencias estadísticamente significativas en las áreas de: dolor corporal, y salud mental, para el grupo con mensaje; y salud general, rol emocional para el grupo sin mensaje.

En la tabla 4, el análisis inter grupos, para el post-test, del cuestionario SB36, evidencia diferencias estadísticamente significativas en el grupo con mensaje en las áreas de: dolor corporal, funcionalidad social, rol emocional, y en el grupo sin mensaje en: salud mental

\section{Discusión}

Se plantea que estudios con diseños verificables (Shama.2011) ofrecen mejores evidencias, donde la intervención musical, contribuye a mejorar, la calidad de vida (CV) de las personas, en sus dimensiones: Siendo el cuestionario SF 36, una opción confiable que muestra que a mayor puntuación, hay mejor estado de salud percibido en las 4 semanas anteriores a su aplicación. Siendo posible estimar los componentes de $\mathrm{CV}$ : físico y mental.

En nuestro estudio, al comparar calificaciones intra e intergrupos, después, de la experiencia, se observó un incremento positivo en las puntuaciones, en la mayoría de las áreas estudiadas de los grupos de mujeres participantes, quienes tuvieron comentarios favorables a la intervención.

Así, la puntuación absoluta: en el grupo con mensajes salutógenos, presentó un incremento promedio de 16.84 puntos, por área; y en el grupo sin mensaje, el 
incremento fue de 12.99 puntos promedio por área, cuya diferencia no fue estadísticamente significativa $(\mathrm{p}=0.57)$.

Cabe señalar que en cuando los incrementos registrados, superan los 5 puntos. Duran, Salazar y Gallegos. (2004), los consideran, clínica y socialmente, significativos.

El estudio, coincide con: Rubia (2009) y, De Oliveira y cols. (2009); que señalan una elevación en las puntuaciones de las variables del cuestionario SF 36, en los grupos que se aplica MT.

Al comparar nuestros resultados con otros, donde solo se aplicó el SF 36, en población mexicana adulta mayor, residente en zonas de extremo desarrollo social y económico del país. Encontramos.:

1.- en Función Física, Rol Físico, y Rol Emocional, nuestros grupos activos con intervención musical, presentan una mayor puntuación, que los reportados en la población de la capital del País (ver tabla 5, Duran..2004), quien también señala que las mujeres, presentan tres veces más riesgo que los varones, de tener mala calidad en la percepción de su funcionamiento físico (Duran..2004). Situación que relaciona a morbilidad y hospitalización en el año previo, Riesgos pensamos que se podrían contrarrestar en el adulto mayor, mediante la realización periódica de ejercicio físico, complementada con MT.

2.- En el área de funcionamiento social las puntuaciones que obtuvieron nuestros dos grupos de intervención, fueron similares al DF, Sonora y Oaxaca (ver Tabla 5), donde se reporta como factor asociado, que estos sujetos tuvieron algún examen médico en el último año, lo que puede aprovecharse la MT, para favorecer el relacionamiento de los adultos mayores con los servicios salud y estos apoyar su ámbito social y emocional.

Debido a que en las dos dimensiones anteriores, las mujeres tienen mayor posibilidad de tener un impacto negativo, sea por eventos de enfermedad, o por el aumento de edad, se requiere elevar su $\mathrm{CV}$, procurando atender las áreas físicas, emocionales y sociales de mujeres que sufren padecimientos crónicos Zanini, Jardim, Salgado, Jardim.(2010),

Así la MT se puede constituir como una opción más a considerar, para mejorar la $\mathrm{CV}$ de la adulta mayor en nuestro medio. 
Por otra parte, las no diferencias estadísticas, en las puntuaciones totales, de los grupos con y sin mensajes salutógenos y música, posiblemente, puedan deberse, al limitado tiempo de exposición del grupo con mensajes para ser totalmente procesados en la conciencia de las adultas mayores, y elevar el puntaje del cuestionario SF36, aunque la música por sí sola, fue un estímulo suficiente para elevar la puntuación total, en ambos casos de intervención.

Sin embargo, son sugestivas de beneficencia, las puntuaciones post test, en el grupo con mensaje en las áreas de dolor, el rol emocional y la funcionalidad social. Acorde a: Kopf.2010 y Chan.2009, quienes señalan que la música al estimular las áreas cerebrales de la motivación y de las, emociones, se provoca mejora en la percepción del dolor, las habilidades de autoestima, interacción y comunicación social (Trailero.2006)(Sanz.1989), señalándose que para que el efecto sea duradero y significativo como MT, requiere ser gestionado conscientemente, desde nosotros mismos, de forma activa y creativa, para lograr resultados más perdurables y de calidad, sobre todo cuando el paciente es capaz de crear su propia música internamente.

Los resultados perfilan a la MT, como una opción de apoyo, en la promoción de salud, en México, para pacientes adultos mayores con enfermedades crónicas, donde la experiencia compartida, puede otorgarle a los actores del proceso salud-enfermedadatención, elementos de sentido para su manejo (Garcia de Alba, Salcedo, Bautista, y Milke..2015)(Salcedo, Garcia de Alba, Velázquez, y Barba.2011) ,situación que ha facilitado la atención de variadas condiciones fisiopatológicas (Chan.2009) (Zanini.2010). Posicionando a la MT como una acción de salud, innovadora estrenada en diversos espacios (hospitalarios, consulta, domicilio), en niños, adultos y adultos mayores, que mejora la CV percibida, e inclusive la relación cuidador-paciente (Ortega, Esteban, Estévez, y Alonso..2015). Situación que las diversas disciplinas del área de área de la salud pueden aprovechar tomando a la MT como medida coadyuvante: Por ejemplo, en el proceso enfermero se ha aplicado para atención y auto atención de pacientes con hipertensión arterial (Chan.2009) (Zanini.2010) donde ha demostrado reducciones estadísticamente significativas en las puntuaciones de depresión, presión arterial y tasa respiratoria, 
Lo anterior plantea a la MT, un futuro prometedor, ajustándose a los cambios y necesidades de la actual atención a la salud donde se ha incrementado en número padecimientos crónicos y discapacidad, en la población adulta mayor, sin dejar de lado a otras poblaciones.

La MT al mejorar la percepción subjetiva, la relación paciente-tratante, o la del paciente-cuidador, en atención cotidiana (De Cunto, y Mendez.2010), y facilitar la verbalización de preferencias musicales después de la audición, posiblemente la persona podrá profundizar reflexivamente su problemática, y compartirnos sus reacciones a la MT y a otros manejos relacionados (Sanz.1989).

\section{Conclusiones}

Los resultados obtenidos, estimulan la continuidad y ampliación de este tipo de estudios, en el manejo multidisciplinario de las adultas mayores . De Oliveira (.2009), que además servirán, para establecer una fluida relación entre ciencias: socio-culturales, de la conducta, y de la salud, que contribuya a erigir un modelo que integre la subjetividad y la objetividad en la cotidianidad (institucional-domestica), del proceso salud-enfermedad-atención; con propósitos preventivos, terapéuticos y de rehabilitación en el ser humano

En resumen, existe un buen número de elementos promotores del bienestar y la percepción de la $\mathrm{CV}$, que están pendientes de ser estudiados, siendo la MT y sus variantes, una opción para futuras investigaciones

Tabla 1

Análisis intra grupo con mensaje pre-postest del cuestionario SF 36.

\begin{tabular}{ccccccccc}
\hline $\begin{array}{c}\text { Grupo } \\
\text { MT con } \\
\text { mensaje }\end{array}$ & $\begin{array}{c}\text { Función } \\
\text { física }\end{array}$ & $\begin{array}{c}\text { Rol } \\
\text { físico }\end{array}$ & $\begin{array}{c}\text { Dolor } \\
\text { Cuerpo }\end{array}$ & $\begin{array}{c}\text { Salud } \\
\text { general }\end{array}$ & Vitalidad & $\begin{array}{c}\text { Función } \\
\text { social }\end{array}$ & $\begin{array}{c}\text { Rol } \\
\text { emocional }\end{array}$ & $\begin{array}{c}\text { Salud } \\
\text { mental }\end{array}$ \\
\hline
\end{tabular}




\begin{tabular}{ccccccccc}
\hline & 67.3 & 33.3 & 43.15 & 43.15 & 49.5 & 57.4 & 53.3 & 57.3 \\
Pretest & \pm & \pm & \pm & \pm & \pm & \pm & \pm & \pm \\
& 17.81 & 5.47 & 2.61 & 2.61 & 14.01 & 12.94 & 0.0 & 6.32 \\
& & & & & & & & \\
Postest & \pm 4.29 & 76.25 & 48.3 & 58.7 & 51.6 & 77.5 & 77.76 & 73.10 \\
& 15.29 & 17.32 & 4.15 & 22.08 & 5.64 & 2.5 & 3.15 & 6.40 \\
Dif abs & 6.99 & 42.95 & 5.15 & 15.55 & 2.1 & 20.1 & 24.46 & 15.8 \\
Dif rel & $9.4 \%$ & $56.3 \%$ & $10.6 \%$ & $26.4 \%$ & $4.06 \%$ & $25.9 \%$ & $31.4 \%$ & $21.6 \%$ \\
$p=$ & 0.61 & 0.17 & $\mathbf{0 . 0 0 0}$ & $\mathbf{0 . 0 1}$ & 0.59 & $\mathbf{0 . 0 0 0}$ & $\mathbf{0 . 0 0 0}$ & $\mathbf{0 . 0 0 0}$ \\
\hline \multicolumn{7}{c}{0} \\
& MT: Músico Terapia; Dif abs: diferencia absoluta; Dif rel: diferencia relativa & &
\end{tabular}

\begin{tabular}{|c|c|c|c|c|c|c|c|c|}
\hline $\begin{array}{l}\text { Grupo MT } \\
\text { sin mensaje }\end{array}$ & $\begin{array}{l}\text { Función } \\
\text { física }\end{array}$ & $\begin{array}{l}\text { Rol } \\
\text { físico }\end{array}$ & $\begin{array}{c}\text { Dolor } \\
\text { Cuerpo }\end{array}$ & Salud general & Vitalidad & $\begin{array}{c}\text { Función } \\
\text { social }\end{array}$ & $\begin{array}{c}\text { Rol } \\
\text { emocional }\end{array}$ & Salud mental \\
\hline Pretest & $\begin{array}{c}66.9 \\
\pm \\
15.96\end{array}$ & $\begin{array}{c}31.6 \\
\pm \\
11.36\end{array}$ & $\begin{array}{c}40.3 \\
\pm \\
4.24\end{array}$ & $\begin{array}{c}52.4 \\
\pm \\
7.52\end{array}$ & $\begin{array}{c}44.9 \\
\pm \\
7.01\end{array}$ & $\begin{array}{c}59.1 \\
\pm \\
17.67\end{array}$ & $\begin{array}{c}60.0^{*} \\
\pm \\
0.0\end{array}$ & $\begin{array}{c}52.3 \\
\pm \\
4.69\end{array}$ \\
\hline Postest & $\begin{array}{c}77.63 \\
\quad \pm \\
12.32\end{array}$ & $\begin{array}{c}69.97 \\
\pm \\
9.90\end{array}$ & $\begin{array}{c}37.65 \\
\pm \\
5.65\end{array}$ & $\begin{array}{c}53.28 \\
\quad \pm \\
17.52\end{array}$ & $\begin{array}{c}54.72 \\
\pm \\
8.03\end{array}$ & $\begin{array}{c}70.8 \\
\pm \\
0.8\end{array}$ & $\begin{array}{c}71.06 \\
\pm \\
3.15\end{array}$ & $\begin{array}{c}76.36 \\
\pm \\
9.99\end{array}$ \\
\hline
\end{tabular}

Tabla 2

Análisis intra grupo sin mensaje pre-postest del cuestionario SF 36. 


\begin{tabular}{ccccccccc} 
Dif abs & 10.7 & 38.37 & 2.65 & 0.88 & 9.82 & 11.7 & 11.06 & 24.06 \\
Dif rel & $13.8 \%$ & $54.8 \%$ & $7.03 \%$ & $1.65 \%$ & $17.94 \%$ & $16.52 \%$ & $15.56 \%$ & $31.5 \%$ \\
$p=$ & $\mathbf{0 . 0 4}$ & $\mathbf{0 . 0 0 0}$ & 0.15 & 0.08 & $\mathbf{0 . 0 0 1}$ & $\mathbf{0 . 0 2}$ & $\mathbf{0 . 0 0 0}$ & $\mathbf{0 . 0 0 0}$ \\
\hline
\end{tabular}

MT: Músico Terapia; Dif abs: diferencia absoluta; Dif rel: diferencia relativa

Tabla 3

Análisis inter grupos pretest del cuestionario SF 36.

\begin{tabular}{|c|c|c|c|c|c|c|c|c|}
\hline Grupo & $\begin{array}{l}\text { Función } \\
\text { física }\end{array}$ & Rol físico & $\begin{array}{l}\text { Dolor } \\
\text { cuerpo }\end{array}$ & Salud general & Vitalidad & $\begin{array}{c}\text { Función } \\
\text { social }\end{array}$ & $\begin{array}{c}\text { Rol } \\
\text { emocional }\end{array}$ & $\begin{array}{l}\text { Salud } \\
\text { menta }\end{array}$ \\
\hline $\begin{array}{l}\text { MT con } \\
\text { mensaje }\end{array}$ & $\begin{array}{c}67.3 \\
\pm \\
17.81\end{array}$ & $\begin{array}{c}33.3 \\
\pm \\
5.47\end{array}$ & $\begin{array}{c}43.15 \\
\pm \\
2.61\end{array}$ & $\begin{array}{c}43.15 \\
\pm \\
2.61\end{array}$ & $\begin{array}{c}49.5 \\
\pm \\
14.01\end{array}$ & $\begin{array}{c}57.4 \\
\pm \\
12.94\end{array}$ & $\begin{array}{c}53.3 * \\
\pm \\
0.0\end{array}$ & $\begin{array}{c}57.3^{*} \\
\pm \\
6.32\end{array}$ \\
\hline $\begin{array}{l}\text { MT sin } \\
\text { Mensaje }\end{array}$ & $\begin{array}{c}66.9 \\
\pm \\
15.96\end{array}$ & $\begin{array}{c}31.6 \\
\pm \\
11.36\end{array}$ & $\begin{array}{c}40.3 \\
\pm \\
4.24\end{array}$ & $\begin{array}{c}52.4 \\
\pm \\
7.52\end{array}$ & $\begin{array}{c}44.9 \\
\pm \\
7.01\end{array}$ & $\begin{array}{c}59.1 \\
\pm \\
17.67\end{array}$ & $\begin{array}{c}60.0 * \\
\pm \\
0.0\end{array}$ & $\begin{array}{c}52.3 \\
\pm \\
4.69\end{array}$ \\
\hline $\begin{array}{c}\text { Dif abs } \\
\text { Dif rel }\end{array}$ & $\begin{array}{c}0.4 \\
0.6 \%\end{array}$ & $\begin{array}{c}1.7 \\
7.2 \%\end{array}$ & $\begin{array}{c}2.85 \\
7.07 \%\end{array}$ & $\begin{array}{c}9.25 \\
17.49 \%\end{array}$ & $\begin{array}{c}4.6 \\
10.2 \%\end{array}$ & $\begin{array}{c}1.7 \\
2.87 \%\end{array}$ & $\begin{array}{c}6.7 \\
11.16 \%\end{array}$ & $\begin{array}{c}5.0 \\
9.56 \%\end{array}$ \\
\hline$p=$ & 0.98 & 0.60 & 0.03 & 0.003 & 0.26 & 0.76 & 0.000 & 0.02 \\
\hline
\end{tabular}

MT: Músico Terapia; Dif abs: diferencia absoluta; Dif rel: diferencia relativa

\begin{tabular}{|c|c|c|c|c|c|c|c|c|}
\hline Grupo & $\begin{array}{l}\text { Función } \\
\text { Física }\end{array}$ & Rol físico & $\begin{array}{l}\text { Dolor } \\
\text { cuerpo }\end{array}$ & Salud general & Vitalidad & $\begin{array}{c}\text { Función } \\
\text { social }\end{array}$ & $\begin{array}{c}\text { Rol } \\
\text { emocional }\end{array}$ & $\begin{array}{c}\text { Salud } \\
\text { mental }\end{array}$ \\
\hline $\begin{array}{l}\text { MT con } \\
\text { mensaje }\end{array}$ & $\begin{array}{c}74.29 \\
\pm \\
15.29\end{array}$ & $\begin{array}{c}76.25 \\
\pm \\
17.32\end{array}$ & $\begin{array}{c}48.3 \\
\pm \\
4.15\end{array}$ & $\begin{array}{c}58.7 \\
\pm \\
22.08\end{array}$ & $\begin{array}{c}51.6 \\
\pm \\
5.64\end{array}$ & $\begin{array}{c}77.5 \\
\pm \\
2.5\end{array}$ & $\begin{array}{c}77.76 \\
\pm \\
3.15\end{array}$ & $\begin{array}{c}73.10 \\
\pm \\
6.40\end{array}$ \\
\hline $\begin{array}{l}\text { MT sin } \\
\text { Mensaje }\end{array}$ & $\begin{array}{c}77.63 \\
\pm \\
12.32\end{array}$ & $\begin{array}{c}69.97 \\
\pm \\
9.90\end{array}$ & $\begin{array}{c}37.65 \\
\pm \\
5.65\end{array}$ & $\begin{array}{c}53.28 \\
\pm \\
17.52\end{array}$ & $\begin{array}{c}54.72 \\
\pm \\
8.03\end{array}$ & $\begin{array}{c}70.8 \\
\pm \\
0.8\end{array}$ & $\begin{array}{c}71.06 \\
\pm \\
3.15\end{array}$ & $\begin{array}{c}76.36 \\
\pm \\
9.99\end{array}$ \\
\hline
\end{tabular}




\begin{tabular}{|c|c|c|c|c|c|c|c|c|}
\hline Dif abs & 3.34 & 6.28 & 10.65 & 5.42 & 3.12 & 6.7 & 6.7 & 3.25 \\
\hline Dif rel & $4.30 \%$ & $8.97 \%$ & $28.28 \%$ & $10.17 \%$ & $5.7 \%$ & $9.46 \%$ & $9.4 \%$ & $4.26 \%$ \\
\hline$p=$ & 0.51 & 0.21 & 0.001 & 0.45 & 0.22 & 0.001 & 0.001 & 0.29 \\
\hline
\end{tabular}

Tabla 4

Análisis Inter grupos postest del cuestionario SF 36.

MT: Músico Terapia; Dif abs: diferencia absoluta; Dif rel: diferencia relativa

\section{Tabla 5}

Calidad de Vida en tres Estados mexicanos, según rubros de SF 36

\begin{tabular}{ccccccccc}
\hline Estado & $\begin{array}{c}\text { Función } \\
\text { física }\end{array}$ & Rol físico & $\begin{array}{c}\text { Dolor } \\
\text { cuerpo }\end{array}$ & Salud general & Vitalidad & $\begin{array}{c}\text { Función } \\
\text { social }\end{array}$ & Rol emocional & $\begin{array}{c}\text { Salud } \\
\text { mental }\end{array}$ \\
\hline Oaxaca & 87.91 & 87.85 & 86.04 & 56.61 & 75.59 & 79.44 & 89.51 & 79.18 \\
Sonora & 90.91 & 89.32 & 85.16 & 48.84 & 67.03 & 71.87 & 88.5 & 66.63 \\
D.F & 63.43 & 40.63 & 68.37 & 48.26 & 59.34 & 73.94 & 47.59 & 62.59 \\
\hline
\end{tabular}

Duran y cols. 2004.(14)

\section{Referencias Bibliográficas}

Benezon, R.O. (2000). Musicoterapia: De Teoría a la Práctica.. Barcelona. Paidos.

Benkovitz, D. (2008). Music therapy and pain management. In: XII Congreso Mundial de Musicoterapia, Anales. Librería Akadia Editorial.2008. Buenos Aires, p.4950

Bruscia, K. (2000). Musicoterapia. (2a ed.). Rio de Janeiro. Enelivros. .

Chan, M. F., Chan, E. A., Mok, E., Tse, K., \& Yuk, F. (2009). Effect of music on depression levels and physiological responses in community-based older adults. International Journal of Mental Health Nursing, 18, (4), 285-294. 
De Cunto-Taets, GG., Mendes-Barcellos. LR. (2010). Música no cotidiano de cuidar: Um recurso terapéutico para enfermagem. Revista de pesquisa: cuidado e fundamental online. 2, (3), 1009-1016

De Oliveira- Zanini, CR. y cols.(2009). El efecto de la musicoterapia en la calidad de vida y en la presión arterial del paciente hipertenso. Arq. Bras.Cardiol. 93, (5), 534-540.

Duran, A L., Salazar, E G., Gallegos, C K. (2004). Estudios sobre la calidad de vida relacionada con la salud del adulto mayor. Citado en: Muñoz, O., García-Peña, C., y Duran, L.. (Eds). La salud del Adulto Mayor, pp: 56-190. Centro Interamericano de Seguridad Social/Instituto Mexicano del Seguro Social. México.

García-de-Alba, J. E. G., Salcedo-Rocha, A. L., Bautista, D. H., \& Milke-Najar, M. E. (2015). Dominio cultural sobre causas de diabetes en tres generaciones de estratos populares en Guadalajara, México. Rev Med Inst Mex Seguro Soc, 53, (3), 308-15

Kopf, A., y Patel, N. B. (2010). Guide to Pain Management in Low-Resource Settings, International Association for the Study of Pain, Seattle: WA. USA.

Maratos, A. S., Gold, C., Wang, X., \& Crawford, M. J. (2008). Musicoterapia para la depresión (Revisión Cochrane traducida). La Biblioteca Cochrane Plus, 2.

Medical Outcomes Trust (2008) SF-36 Health Survey Update. Recuperado de http://www.sf-36.org/ Recuperado de: http://dgpldes.salud.gob.mx DOI:10.1007/978-3-540-29805-2_2338

Myskja, A. (2008). Integrated music-an approach to improved health and wellbeing in nursing homes. XII Congreso Mundial de Musicoterapia, Anales. Librería Akadia Editorial. 2008. Buenos Aires, p.400-401.

Ortega, E., Esteban, L., Estévez, A. F., \& Alonso, D. (2015). Aplicaciones de la musicoterapia en educación especial y en los hospitales. European Journal of Education and Psychology, 2015. 2. (2).145-168

Radulovic, R., Cvetkovic, M., \& Pejovic, M., (November.1997). Complementary musical therapy and medicamentous therapy in treatment of depressive disorders. In WPA Thematic Conference Jerusalem. November 1997.

Rubia-Vila, F.( 16 deAbril.de 2009). Música y Cerebro. Conferencia llevada a cabo en la Real Academia Nacional de Medicina. Madrid. España,

Sanz, E. G. (1989). Musicoterapia y enriquecimiento personal. Revista interuniversitaria de formación del profesorado. (4), 91-108. 
Salcedo-Rocha, A. L. García-de-Alba-Garcia, J. E. Velásquez-Herrera, J. G. \& BarbaGonzález, E. A. (2011). Oral Health: Validation of a questionnaire of selfperception and self-care habits in Diabetes Mellitus 2, hypertensive and obese patients. The UISESS-B scale. Med Oral Patol Oral Cir Bucal, 16, (6), 834-9.

Sharma, M., \& Mathur, K. C. (2011). Effects Of Music Therapy On Clinical And Biochemical Parameters Of Metabolic Syndrome. Journal of Bangladesh Society of Physiologist. 6, (2), 108-115.

Trallero Flix C. (2006). Tratamiento del estrés docente y prevención del burnout con musicoterapia auto realizadora. Revista virtual Psiquiatria.com. Recuperado de:http://www.psquiatria.com/congreso/2006/estres/articulos/23916/

Zanini, C. Jardim, P. C. Salgado, C. \& Jardim, T. (2010). Music therapy contributing to the quality of life of hypertensive patients . Journal of Hypertension: 28, (6), e540 